0-10). Utilities were measured with the EuroQoL (EQ-5D $\left.D_{\text {utility }}\right)$ (range 0-1). A time-integrated summary score defined the clinical effects (BASFI-AUC) and utilities (EQ-5D $\mathrm{D}_{\text {utility }}$ AUC) over time. Both direct (health care and non-health care) and indirect costs were included. Resource utilisation and absence from paid work were registered weekly by the patients in a diary. All costs were calculated from a societal perspective.

Results 111 patients completed the diary (group $1 \mathrm{n}=38$; group $2 \mathrm{n}=36$; control group $\mathrm{n}=37$ ). The between-group difference $(95 \% \mathrm{CI})$ for the BASFI-AUC was $1.0(0.4$ to 1.6 ; $\mathrm{p}$ $=0.001)$ for group 1 versus controls, and $0.6(0.1$ to $1.1 ; \mathrm{p}=$ $0.020)$ for group 2 versus controls. The between-group difference for the EQ-5D $\mathrm{D}_{\text {utility }}$-AUC was 0.17 (0.09 to $0.25 ; \mathrm{p}<$ $0.001)$ for group 1 versus controls, and 0.08 (0.00 to $0.15 ; \mathrm{p}=$ 0.04) for group 2 versus controls. The mean total costs per patient (including costs for spa therapy) during the study period were ?3023 for group 1, ?3240 for group 2, and ?1754 for the control group. The incremental cost-effectiveness ratio was ? 1269 per unit effect gained in functional ability for group 1, and ?2477 for group 2. The costs per QALY gained were ?7465 for group 1 and ?18575 for group 2 .

Conclusion Combined spa-exercise therapy is more effective and shows favourable cost-effectiveness and cost-utility ratios compared to weekly group physical therapy alone in patients with ankylosing spondylitis.

\section{OP0055 SULFASALAZINE IMPROVES THE NUMBER OF FLARES OF ACUTE ANTERIOR UVEITIS OVER A ONE YEAR PERIOD}

${ }^{1} S$ Muñoz-Fernández, ${ }^{2} V$ Hidalgo, ${ }^{1} G$ Bonilla, ${ }^{1}$ J Fernández, ${ }^{2} A$ Schlincker, ${ }^{2} A B$ Larqué, ${ }^{2}$ A Fonseca, ${ }^{1} E$ Martín-Mola. ${ }^{1}$ Rheumatology; ${ }^{2}$ Ophthalmology, Hospital Universitario LA PAZ, Madrid, Spain

\subsection{6/annrheumdis-2001.347}

Background Sulfasalazine is used in RA, but its role in uveitis is not well established.

Objectives To study the efficiency of sulfasalazine (SSZ) in the treatment of recurrent flares of acute anterior uveitis (AAU) over a one year period.

Methods From June of 1997 to October 2000, 376 patients with uveitis have been attended by Ophthalmologists and Rheumatologists in our outpatient Uveitis Unit according to a protocol based on the pattern of uveitis and clinical symptoms of the patient. Data were prospectively recorded. The criteria to start treatment were: 1) The presence of three or more flares of AAU in the previous year or 2) Recurrence of the uveitis in less than three months from the previous episode. Exclusion criteria to start SSZ were uveitis due to infectious or malignant causes or the presence of any contraindications to this drug. SSZ was started at $500 \mathrm{mg}$ per day and gradually increased untill a dose of $2 \mathrm{~g}$ per day. If inadecuate response, the dose was increased untill $3 \mathrm{~g}$. The major outcome was defined as the number of flares of uveitis over a one year period compared in the same group of patients with the flares of the previous year without SSZ, followed prospectively in our unit. Wilcoxon test was used for the statistical study.

Results Eleven patients were in SSZ treatment $(5$ men, 6 women; 6 ankylosing spondylitis 2 idiopatic uveitis, one Reiter's syndrome, one intestinal inflammatory disease and one juvenil chronic arthritis). Three of them started SSZ for other reasons than uveitis or in other services of the hospital and were then excluded of the analysis. In the remaining 8 patients, the mean number of AAU flares in the pre-SSZ year was 3.5 (ranging from 3 to 4 flares, SD 0.5). After a one year of treatment, the mean of flares was reduced to 0.6 (ranging from 0 to 2 flares, SD 0.9$)$ that was found to be significant $(p=0.011)$. Only one patient was found to be a non-responder. Other two patients were considered partially responderes with a reduction from 4 to 2 and from 3 to 1 flares respectively in comparison of the pre and post-treatment one year period. The other 6 patients did not have any new flare of uveitis during the one year SSZ treatment. We found only two adverse events, both with increased transaminase profiles that did not result in discontinuation of the therapy.

Conclusion SSZ treatment reduce the number of flares over a one year period in patients with recurrent AAU.

\section{OP0056 TNF-ALPHA BLOCKADE WITH INFLIXIMAB IN PATIENTS WITH ACTIVE SPONDYLOARTHROPATHY: FOLLOW-UP OF ONE YEAR MAINTENANCE REGIMEN}

E Kruithof, F Van den Bosch, D Baeten, F De Keyser, H Mielants, EM Veys. Rheumatology, Ghent University Hospital, Ghent, Belgium

\subsection{6/annrheumdis-2001.348}

Background In an open pilot study, the anti-TNF-alpha monoclonal antibody, infliximab, induced a rapid and significant improvement in global, peripheral and axial disease manifestations of patients (pts) with active spondyloarthropathy (SpA), without major side effects. ${ }^{1}$

Objectives The aim of this study was to determine whether repeated infusions of infliximab would effectively and safely maintain the observed effect.

Methods The safety and efficacy of a maintenance regimen (5 $\mathrm{mg} / \mathrm{kg}$ IV infliximab every 14 weeks) was evaluated using the same measurements reported in the open label study. ${ }^{1}$ Of the initial 21 pts 19 completed the 1 year follow-up for efficacy. Two pts (1 psoriatic arthritis, 1 undifferentiated $\mathrm{SpA}$ ) changed to another dosing regimen of infliximab after week 12 due to partial lack of efficacy. However, these pts are still in follow-up and are included in the safety analysis.

Results Efficacy: After every retreatment (at week 20, 34 and 48) a sustained significant decrease of global, peripheral and axial disease manifestations was observed $(\mathrm{p}<0,05$ compared to baseline). Before retreatment, recurrence of symptoms was observed in 3 pts $(16 \%)$ at week 20, 13 pts $(68 \%)$ at week 34 and 15 pts $(79 \%)$ at week 48 . Safety: No withdrawals due to adverse events occurred. No significant laboratory abnormalities were detected. No major peri-infusional allergic reactions were observed. Three infectious episodes (pyelonephritis, otitis media, and tooth abscess) were observed. During the 1 year follow-up 12 pts (57\%) developed on at least 2 occasions antinuclear antibodies; in 4 of these pts (19\%) antibodies to dsDNA were detected. However, no lupus-like syndromes occurred.

Conclusion In this open follow-up study of infliximab in pts with active $\mathrm{SpA}$, the significant improvement of all disease manifestations was maintained over a 1 year follow-up period without major adverse events. Since recurrence of symptoms was observed in a rising number of patients with every retreatment, the maintenance regimen $(5 \mathrm{mg} / \mathrm{kg}$ every 14 weeks) probably needs adjustment (shorter interval or higher dose).

\section{REFERENCE}

1 Ann Rheum Dis. 2000;59:428-33 


\section{OP0057 THE PATIENTS' KNOWLEDGE ABOUT THEIR DISEASE: A FRENCH MULTICENTRIC SURVEY IN ANKYLOSING SPONDYLITIS (AS)}

P Claudepierre, RM Flipo, J Sibilia, JM Berthelot, P Goupille, S Cortinovis, X Chevalier, D Wendling. Rheumatology, Henri Mondor Hospital, Créteil, France

\subsection{6/annrheumdis-2001.349}

Background It is known that, in chronic diseases, patient education is a factor of good compliance and good disease management. An index was recently proposed to assess the level of knowledge in AS. ${ }^{1}$

Objectives To assess the level of knowledge of a population of AS patients from different tertiary care centres in France, and to identify some factors linked with this level.

Methods Study:multicentric cross-sectional study. No structured educational program was used in these centres at the time of the study. Patients: Inclusion criteria were New York criteria of AS and patients had to read French fluently. Data collected: demographic and sociocultural, disease characteristics, the index of knowledge in a French version (obtained from a double translation) giving a maximum score of 25 . Statistical analysis: to seak a link between the knowledge score and collected factors, usual non parametric tests were used.

Results The mean score of the 95 included patients was only 16.4 (S. D. 4.8). The percentages of correct answers were not equally distributed among the 4 groups or domains of questions. The duration of disease management in a tertiary care centre appeared to be positively linked to the level of knowledge $(r=$ 0.28 with $\mathrm{p}=0.01$, for the global score on 25 points, and $\mathrm{r}=$ 0.26 with $\mathrm{p}=0.02$, for the number of good answers (on 14) per patient). It was also observed that the level of knowledge was better in females than males, and in patients who have previously read on the disease or who knew the association of AS patients. No interaction was found between the factor "sex" or the factor "duration of disease management in a tertiary care centre" and the other identified factors, suggesting that they are independent factors associated with the level of knowledge. Conversely, the 3 other factors, e.g., level of education in 2 classes, previous reading, knowing of the association, were statistically linked two by two. The other tested factors were not significantly linked with the level of knowledge: age of the patients, duration of the disease, characteristics of the disease (axial or peripheral, psoriasis, radiological severity).

Conclusion The level of knowledge of these patients appears lower than this observed in the previous UK study, reinforcing the positive role of educational programs. These data will help to determine in which domains and in which patients the highest effort of education should be initially done.

\section{REFERENCE}

1 Lubrano E, et al. Br J Rheumatol. 1998;37:437-41

\section{OP0058 EFFICACY OF INFLIXIMAB IN SEVERE REFRACTORY ANKYLOSING SPONDYLITIS (AS). RESULTS OF AN OPEN- LABEL STUDY}

${ }^{1} \mathrm{MA}$ Breban, ${ }^{2} \mathrm{E}$ Vignon, ${ }^{3} \mathrm{P}$ Claudepierre, ${ }^{4} \mathrm{~A}$ Saraux, ${ }^{5} \mathrm{D}$ Wendling, ${ }^{6} \mathrm{E}$ Lespesailles, ${ }^{7} \mathrm{~L}$ EullerZiegler, ${ }^{8} \mathrm{~J}$ Sibilia, ${ }^{9} \mathrm{~A}$ Perdriger, ${ }^{10} \mathrm{C}$ Alexandre, ${ }^{1} \mathrm{M}$ Dougados. ${ }^{1}$ Rheumatology Division, Cochin Hospital, Paris; ${ }^{2}$ Rheumatology Division, Lyon; ${ }^{3}$ Rheumatology Division, Créteil; ${ }^{4}$ Rheumatology Division, Brest; ${ }^{5}$ Rheumatology Division, Besançon; ${ }^{6}$ Rheumatology Division, Orléans; ${ }^{7}$ Rheumatology Division, Nice; ${ }^{8}$ Rheumatology Division, Strasbourg; ${ }^{9}$ Rheumatology Division, Rennes; ${ }^{10}$ Rheumatology Division, Saint-Etienne, France

\subsection{6/annrheumdis-2001.350}

Background Early reports have emphasised the efficacy of the anti-TNF-alpha monoclonal antibody, infliximab, to treat spondylarthropathy patients. Further studies are required to evaluate the extent of such efficacy in large subgroups of well-defined patients.

Objectives To examine the effect of infliximab, in severe AS with predominant axial symptoms.

Methods Patients suffering of severe AS, with evidence of systemic inflammation (modified New-York criteria; BASDAI $>=$ 30/100; CRP $>=15 \mathrm{mg} / \mathrm{l})$ ), and without present peripheral arthritis or extraskelettal manifestation were included. Previous DMARD's had to be discontinued for $>=3$ months. Intake of NSAID had to be stable. Eligible patients received 3 infusions of infliximab $(5 \mathrm{mg} / \mathrm{kg}$, weeks 0,2 and 6). Clinical and biological monitoring was planned for 6 months.

Results We enrolled 48 parents $(36 \mathrm{M} / 12 \mathrm{~F}$; mean age $=36 \pm 9$ yr); 41/46 (89\%) were HLA-B27+; 43 (90\%) completed the treatment, as scheduled, whereas 5 received only 2 infusions. Interim result (wk 8) is shown, and compared to baseline in the table. Altogether, 44 patients (92\%) were responders, as defined by a reduction of global pain on VAS of at least $20 \%$, as compared to baseline level.

\begin{tabular}{lllll}
\multicolumn{5}{l}{ Abstract OP0058 Table 1 } \\
\hline & Baseline & Week 8 & Variation (\%) & P value \\
\hline Disease activity (VAS) & $70 \pm 21$ & $18 \pm 21$ & $-74 \%$ & $<0.0001$ \\
Globlal pain (VAS) & $67 \pm 22$ & $16 \pm 2$ & $-75 \%$ & $<0.0001$ \\
BASDAI & $57 \pm 16$ & $16 \pm 14$ & $-73 \%$ & $<0.0001$ \\
BASFI & $61 \pm 21$ & $19 \pm 2$ & $-70 \%$ & $<0.0001$ \\
CRP (mg/L) & $50 \pm 31$ & $8 \pm 11$ & $-85 \%$ & $<0.0001$ \\
\hline
\end{tabular}

Conclusion Our data confirm the remarkable efficacy of infliximab in AS. It indicate that most, patients suffering of inflammatory active AS promptly and dramatically respond to this treatment.

\section{OP0059 INFLIXIMAB IN REFRACTORY SPONDYLOARTHROPATHIES; PRELIMINARY RESULTS IN A SPANISH POPULATION}

${ }^{1} \mathrm{E}$ Collantes, ${ }^{1} \mathrm{MC}$ Muñoz-Villanueva, ${ }^{2} \mathrm{R}$ Sanmarti, ${ }^{2} \mathrm{JD}$ Cañete, ${ }^{3} \mathrm{~J}$ Gratacos, ${ }^{4} \mathrm{P}$ Zarco, ${ }^{5} \mathrm{C}$ Gonzalez, ${ }^{6} \mathrm{JC}$ Torre. ${ }^{1}$ Rheumatology, Hospital Universitario Reina Sofia, Cordoba; ${ }^{2}$ Rheumatology, Hospital Clinic, Barcelona; ${ }^{3}$ Rheumatology, Hospital Parc Tauli, Sabadell; ${ }^{4}$ Rheumatology, Fundacion Hospital, Alcorcon; ${ }^{5}$ Rheumatology, Hospital Gregorio Marañon, Madrid; ${ }^{6}$ Rheumatology, Hospital Monte Naranco, Oviedo, Spain

10.1136/annrheumdis-2001.351

Background Infliximab, a chimeric anti-TNF-alpha antibody has been used successfully for the treatment of rheumatoid arthritis 